\title{
The visual field and visually guided behavior in the zebra finch (Taeniopygia guttata)
}

\author{
Hans-Joachim Bischof \\ Universität Bielefeld, Fakultät für Biologie, Postfach 8640, D-4800 Bielefeld 1, Federal Republic of Germany \\ Accepted February 4, 1988
}

Summary. Measurements were made of the physical properties of the visual system of the zebra finch, a bird with laterally placed eyes. The use of the visual system in pecking and courtship behavior was examined. It was demonstrated that the optical axis and the fovea of the eye point in a direction about $62^{\circ}$ from the sagittal axis of the head. The visual field of each eye covers about $170^{\circ}$ in the horizontal plane. In the frontal region there is an overlap of about $30^{\circ}-40^{\circ}$ where the birds can see binocularly; caudally there is a 'gap' in the visual field of $60^{\circ}$. The point of best binocular viewing is in the sagittal plane at $16.5^{\circ}$ below the beak.

Concerning movement detection, the upper threshold is $540^{\circ} / \mathrm{s}$ for the binocular (frontal) part of the visual field and about $1100 \%$ s for the monocular (lateral) part. Most fixations before pecking occur monocularly. A preference for one eye during pecking was not detected. During the courtship song, a male bird directs its head towards the female. The results are discussed in comparison with findings in pigeons and chickens.

\section{Introduction}

Although the behavior of no other class of vertebrates is as dependent on visual cues as that of birds, quantitative studies on visually guided behavior in these animals are rare. Most of the studies on birds are concerned with the anatomy of the visual system (reviewed in Meyer 1977; Donovan 1978) or the neurophysiology of vision (reviewed in Emmerton 1983a). Others deal with psychophysical functions or discrimination learning (reviewed in Emmerton 1983b).
Moreover, with a few exceptions (e.g., Friedman 1975), most studies have been performed with pigeons, so there is almost no basis for a comparative study of visually guided behavior in birds. For this reason morphological and behavioral features of visually guided behavior were examined in the zebra finch, a bird which has recently become a standard laboratory animal.

Zebra finches are small, dimorphic, grain-eating birds. In common with most avian species their eyes are placed laterally. However (see Fig. 1), a certain binocular overlap can already be seen by inspection. The use of binocular information in pecking behavior is very well documented in the pigeon. Several studies indicate that the pigeon fix-

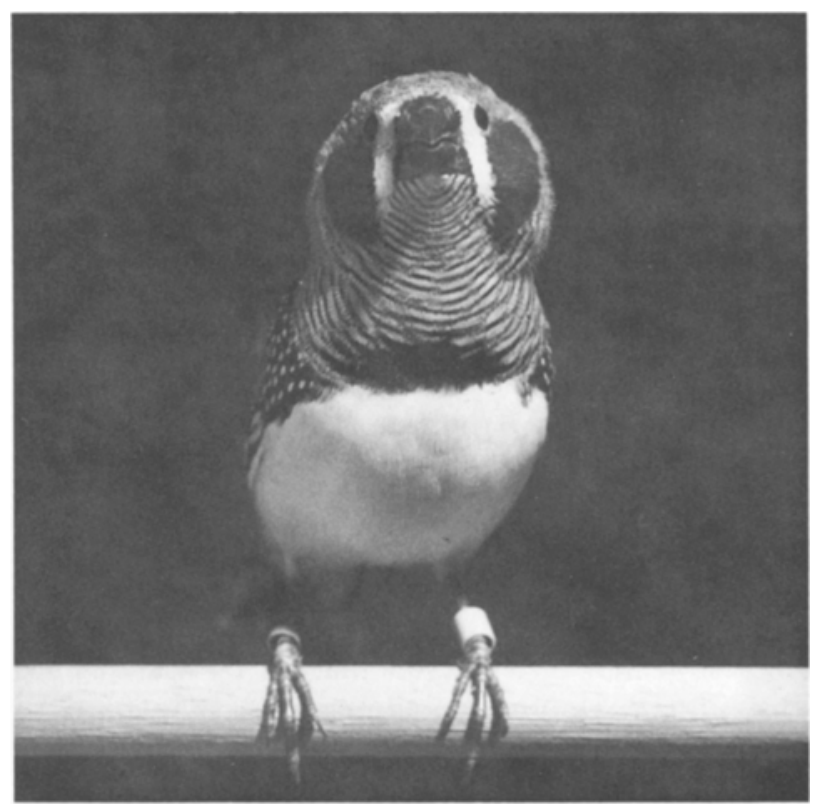

Fig. 1. Male zebra finch, photographed from slighty above the point of best binocular vision. For explanation see text 
ates the seed binocularly before it starts pecking. Martinoya et al. (1984) demonstrated that the eyes of the bird converge while the head is moving towards the grain. Goodale (1983) found that pigeons have two points at which they fixate before pecking occurs. At the first point, at a distance of about $8 \mathrm{~cm}$ from eye to grain, the head is held still for about $0.5 \mathrm{~s}$, during which time the bird decides which grain shall be eaten. At the second point the distance of the grain and its size is estimated. From this point on, the head movement is ballistic and not entirely under visual control, as the eyes are closed when the bird hits the grain.

Friedman (1975) found that in barbary doves the grain is often fixated monocularly before pecking. In this species, the proportion of monocular vs. binocular field used in fixation depends on the distance of the grain from the eye. Although frontal and lateral fixation occurs independently of grain distance, more fixations are performed laterally and fewer frontally when the bird's head is further away.

Observing pecking behavior in zebra finches, my first impression was that in nearly all cases they used monocular fixation before pecking. To examine this more quantitatively, Friedman's method (1975) was used. As nothing is known about the structure of the visual field of zebra finches, some measurements were made to determine which sections were binocular and which were monocular, and the point of best binocular viewing was calculated.

Some morphological data concerning the placement of the eyes and the retinal specializations is also presented. To evaluate physiological differences between the processing of information in the frontal and lateral parts of the visual field, the influence of frontal or lateral eye covers on the ability of the birds to detect and to respond to fast movements was investigated.

Some authors have suggested that pigeons, and perhaps birds in general, use binocular cues (frontal vision) in pecking, whereas the more distant environment is, in most cases, registered monocularly (lateral vision; e.g., Bloch and Martinoya 1982). In order to distinguish between these possibilities my students and I decided to study the frontal-lateral viewing preferences in another kind of behavior, the courtship behavior of male zebra finches, which is triggered mainly by visual cues (Bischof 1985). Normal courtship is a chain in which each behavioral act is triggered by the previous behavior of the partner (Morris 1954; Immelmann 1959). As each bird has to detect the signal stimuli given by the partner over the entire period of the courtship sequence, courtship behavior should be under visual control. If the abovedescribed distinction between the relative contribution of lateral and frontal viewing is true, the birds should perform this control monocularly. In fact, a recent report from Workman and Andrew (1986) demonstrates that male zebra finches preferentially use the right eye to fixate the female between courtship bouts.

\section{Material and methods}

The experiments described below were performed with adult zebra finches of both sexes from the institute's stock. All birds were housed in individual cages during the whole experiment.

Anatomical features. For a rough anatomical inspection of the position of the eyeballs within the orbits a zebra finch was decapitated after deep Nembutal anesthesia and the head was immediately sectioned in a horizontal plane through the middle of the eyeballs. The plane was parallel to the inner edges of the upper and the lower bill (Fig. $2, \alpha=0^{\circ}$ ). Therefore, the cut was also nearly horizontal with respect to the normal head position of zebra finches (unpublished results). For examination of the retina, five birds were, after deep anesthesia, perfused intracardially with physiological saline followed by $10 \%$ formaldehyde; the eyes were then removed, embedded in paraffin and cut in the horizontal plane. The sections were stained with Cresyl violet.

Measurement of the visual field. For estimation of the visual field of the bird the method of Martinoya et al. (1981) was adopted. Using this method, the bird's head was fixed in its normal position by clamping a small plastic screw, which had been cemented onto the skull, into a device allowing $360^{\circ}$ horizontal rotation of the bird (plane $\beta$ - azimuth - in Fig. 2). A dissecting microscope with its optical axis placed horizontally was used to measure the pupil (magnification $160 \times$ ). In three birds the horizontal and vertical axes of the left and/or right pupil were measured with the help of the ocular scale of the

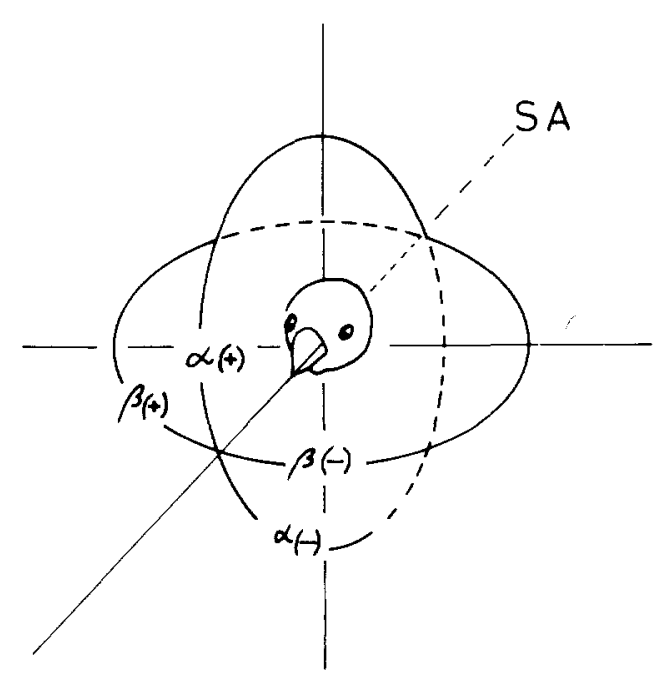

Fig. 2. Coordinate system used in this study. $S A$, sagittal axis; $\beta$, azimuth; $\alpha$, elevation 
microscope in steps of $10^{\circ}$. As zebra finches can move the eyes independently of the head, to ensure that the bird looked towards the microscope a stuffed female dummy was presented just below the microscope optics. In all cases this caused the bird to look in the direction of the microscope. A pupillary index was calculated by dividing the horizontal diameter of the pupil by the vertical diameter.

Point of best binocular vision. The point of best binocular vision was defined by Martinoya et al. (1981) as the position in the visual field from which the receptive fields of both eyes have the best overlap. In the horizontal plane, this must be at an angle $\beta$ of $0^{\circ}$ (see Fig. 2). In the vertical plane $(\alpha-$ elevation; Fig. 2), Martinoya et al. (1981) suspected that the best overlap of the visual fields would be at a point where the long axes of the pupils of both eyes, which from the front of the animal appear as ellipses, are parallel $\left(\gamma=0^{\circ}\right)$. For estimation of the point of best binocular viewing, five birds were again fixed according to the method described above, and the microscope was moved round the head of the birds in the sagittal plane $\left(\beta=0^{\circ}\right)$. The angle between the long axes of both pupils $(\gamma)$ was measured every $10^{\circ}(\alpha)$ (Fig. 5 ).

Upper threshold of movement detection. Moving stripe patterns induce a following response movement of the head in birds; this is called head nystagmus as the movement pattern is very similar to the eye movements induced by moving gratings. The influence of the velocity of movement on the head nystagmus was observed in birds which were placed inside a rotating drum with a diameter of $40 \mathrm{~cm}$. The inner walls were covered with 2-cm-wide alternating black and white stripes. The body of the bird was fixed by wrapping it in a piece of cloth, leaving the head free. The illumination (12-V DC source) was held constant at 1500 lux as measured at the bottom of the rotating drum. The velocity of the striped pattern was estimated by measuring the frequency of the black-white cycles with an optical device and calculating the velocity $(\% / s)$. The optokinetic head nystagmus was monitored by a video camera mounted on the top of the experimental apparatus. Lateral and frontal viewing were prevented by fixing small pieces of black tape onto the bird's head in either the frontal or lateral field of view. This treatment disturbed the birds only for a few minutes.

The upper velocity threshold of movement detection was determined by raising the speed of rotation of the drum from zero until the head nystagmus disappeared. After each trial the drum was stopped and the next trial was started 2 min later. About 20 trials were performed in one session (the session was finished earlier if the bird showed signs of stress).

Pecking and sexual behavior. For a demonstration of the pecking sequence (Fig. 8) a small compartment $(10 \times 10 \times 10 \mathrm{~cm})$ with a perch and a small feeder was constructed. The sides and the top of this compartment were made of glass and a mirror was mounted at the top of the cage at an angle of $45^{\circ}$. This construction allowed the feeding behavior of the birds to be recorded simultaneously from the top and from the side.

For the analysis of pecking and courtship behavior a cage of $50 \times 50 \times 50 \mathrm{~cm}$ was constructed. The front and top of the cage were made of glass. A mirror was placed on the top at an angle of $45^{\circ}$. With the help of this mirror, pecking and courtship behavior could be recorded from above by a video camera placed in front of the cage. Grains were randomly distributed over the floor.

Pecking behavior was studied by analyzing the video images frame by frame during successive pecks. The fixation phase was easy to determine because the image of the bird's head did not alter for at least two or three pictures. At this position, the angle between the sagittal midline of the head and a line drawn from the grain through the middle of the eye of the bird was measured. If the grain was in a position in front of the head such that this line was parallel to the midline or the resulting angle was negative (meeting the midline in front of the head), the fixation was rated as binocular (see Results). These measurements were performed in three birds. In addition, the horizontal distance between grain and eye in each pecking sequence was measured. In eight birds (including the three mentioned above) the frequencies of left and right eye fixations during an experiment were calculated by observing the behavior of the birds in slow motion. For observation of courtship sequences, a male and a female which had been isolated for at least 3 days before the experiment were introduced into the cage. The behavior of the birds was recorded from above and fixation was analyzed during bouts of male courtship song using a similar technique to that used in the analysis of pecking. In this case the angle between the sagittal axis of the head of the male and the line between the male's eye and the middle of the female's head was measured.

\section{Results}

\section{Anatomical features}

Figure 3 is a schematic representation of the horizontal section of the 'nonfixated' head. It shows that the eyes of the zebra finch are large enough to meet in the midline of the head. The optical axis $(\mathrm{OA})$ is at an angle of approximately $62^{\circ}$ from the sagittal plane; the fovea $(\mathrm{F})$ is slightly more frontal and the pecten $(\mathrm{P})$ is lateral to the meeting point between the optical axis and the retina. Figure 4 shows that the birds have a very well developed deep fovea. It is not known whether there is a second area of higher cellular density, like the red area in the pigeon, which can be detected by eye.

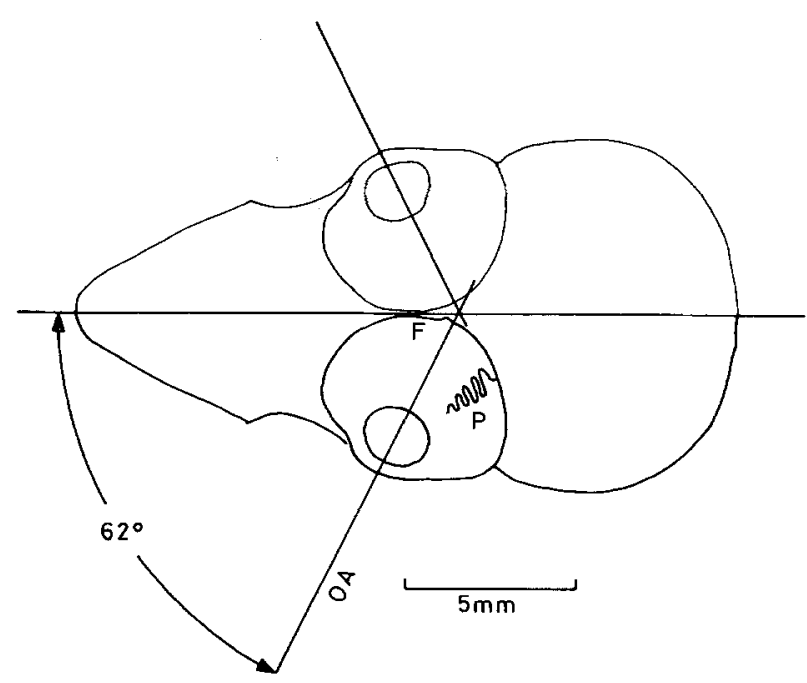

Fig. 3. Position of the eyes within their orbits. $F$, fovea; $P$, pecten; $O A$, optical axis 


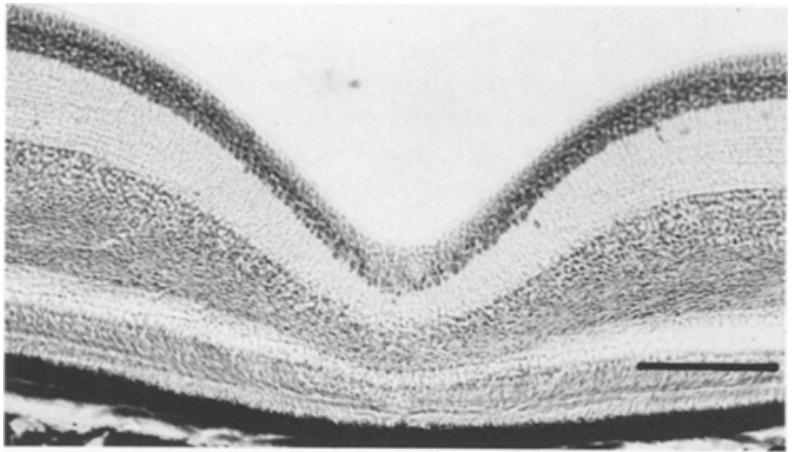

Fig. 4. Horizontal section of the foveal area of the right eye. $30 \mu \mathrm{m}$ sections, cresyl violet stain. Bar, $100 \mu \mathrm{m}$

\section{Monocular and binocular fields}

The visual field of the zebra finch at an elevation of $0^{\circ}(\alpha$, Fig. 2$)$ was measured by a method developed by Martinoya et al. (1981). Figure 5 shows the results (means of measurements from three birds). The highest pupillary index of 0.95 (the horizontal axis of the pupil is almost as long as that of the vertical axis) is at about $50^{\circ}-60^{\circ}$ from the midline, which is consistent with the morphological measurements of the optical axis (Fig. 3). The diagram also shows that there is an overlap of the two fields of approximately $30^{\circ}$. The $20^{\circ}$ value of the pupillary index is 0 , i.e., the horizontal diameter of the eye at that angle is zero. Therefore, stimuli at angles more than about $15^{\circ}-20^{\circ}$ from the midline cannot be detected binocularly. Behind the head there is a gap of about $60^{\circ}$ where the zebra finch cannot see, the 'blind area'.

\section{Point of best binocular vision}

The angle between the long axes of the pupils of both eyes $(\gamma)$ was measured (Fig. 6) for every $10^{\circ}$ in the sagittal plane ( $\alpha$, Fig. 2). Each point represents the mean of ten measurements in one bird. The line connects the means of the five points of each $\alpha$ position and meets the $\mathrm{x}$-axis at $-16.5^{\circ}$. At this point, $16.5^{\circ}$ below the direction of the beak (SA, Fig. 2), the long axes of the two pupils are parallel, this being the point of best binocular viewing as defined by Martinoya et al. (1981). This value is in accordance with the results obtained from pigeons (Martinoya et al. 1981).

\section{Movement sensitivity}

The results from the optokinetic head nystagmus experiment are shown in Fig. 7. About 100 measurements were made in three birds with the frontal

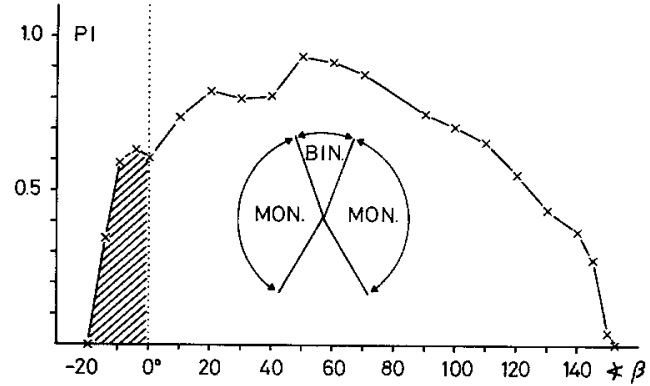

Fig. 5. Visual field of the zebra finch. Measurements in the horizontal plane $(\beta)$. $P I$, pupillary index; shaded area, binocular overlap. Inset, schematic drawing of the visual field; bin, binocular area; mon, monocular area

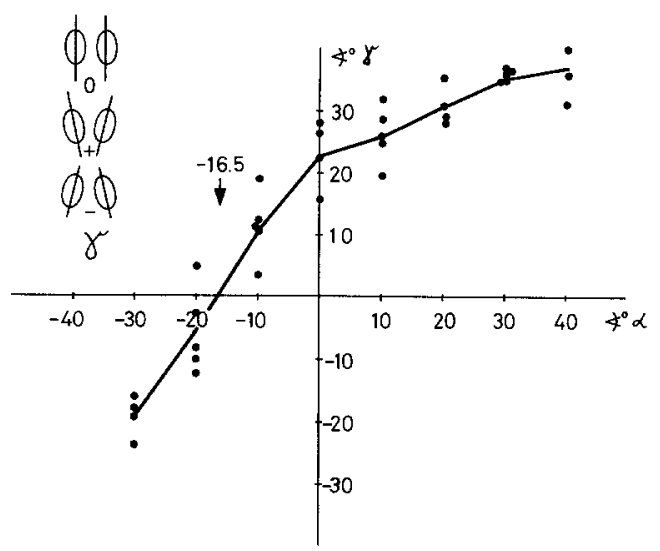

Fig. 6. Direction of best binocular viewing (see text). Each point represents the mean of the measurements of one bird. The continuous line connects the means of all measurements for each angle $\alpha$ (elevation, see Fig. 2). Inset, definition of $\gamma$, the angle between the long axis of the two eye images

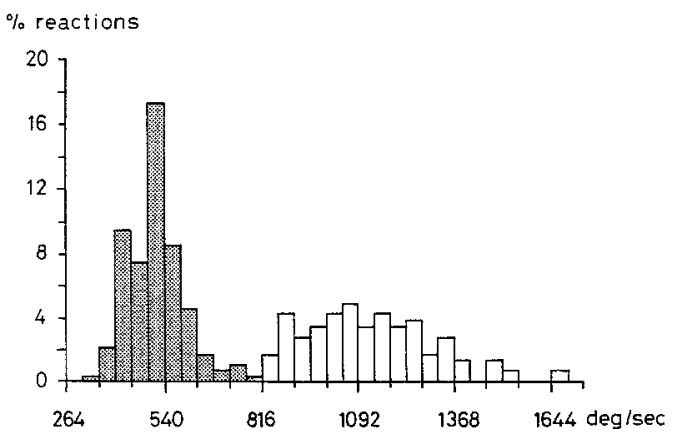

Fig. 7. Upper velocity detection threshold with exposed binocular field (stippled bars) and monocular field (open bars). Bin width $46^{\circ} / \mathrm{s}$

visual field covered (monocular field, open bars) or with the lateral visual field covered (binocular field, hatched bars). With frontal vision the measurements at the high velocity threshold are distributed around $520^{\circ} \mathrm{s}$; in lateral viewing the mean 


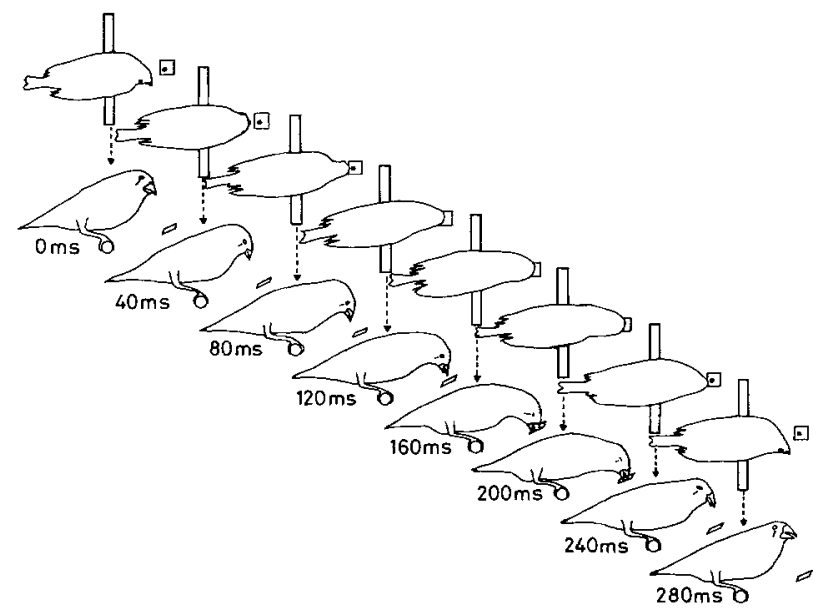

Fig. 8. Pecking sequence of a zebra finch. For explanation see text

value is nearly doubled. Moreover, an overlap between both distributions can be observed only in the $770^{\circ} / \mathrm{s}$ to $816^{\circ} / \mathrm{s}$ bin. This demonstrates that the bird's ability to detect fast movements is much better with the lateral visual field than with the frontal field.

\section{Pecking behavior}

Figure 8 is a schematic drawing of a pecking sequence in a bird sitting on a perch and feeding from a feeder (see Methods). With this setup it was possible to record the behavior of the bird from above and from the side simultaneously. Although the situation in this experiment is different from that in the experiments in which the fixation angles were measured (where the birds sat on and fed from the floor), the overall pattern of fixation and pecking is similar in both cases, as shown by qualitative comparisons. The upper row of the figure is a view from the top, and the lower row a side view of the bird. The first picture represents the end of the fixation period, where the bird looks at the grain monocularly. The next pictures show the movement of the head towards the grain. Between 120 and $160 \mathrm{~ms}$ from the beginning of the movement, the bird closes its eyes and opens its beak. The grain is grasped at $200 \mathrm{~ms}$. It could not be determined from the recordings whether a second fixation period occurs at this point, as the low time resolution of the video equipment $(40 \mathrm{~ms})$ did not allow detection of short head stops. However, the observations of the pecking sequence demonstrate that the overall pattern of pecking in zebra finches is similar to that observed in pigeons.

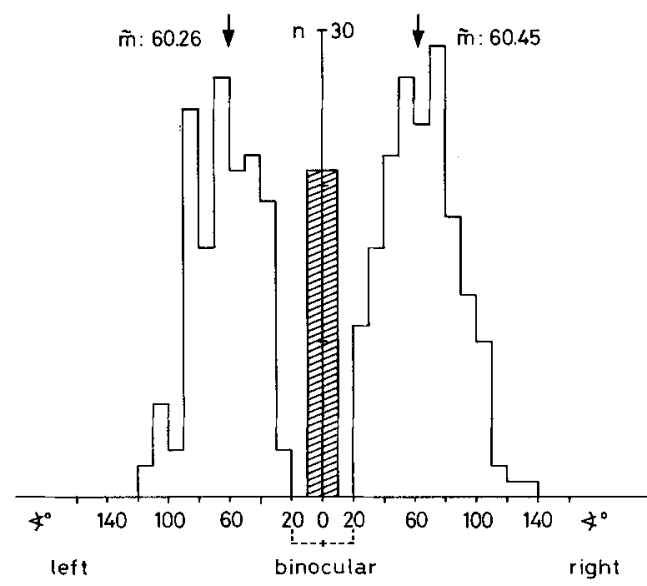

Fig. 9. Angles of fixation before pecking measured from the midline of the head. Measurements only in the horizontal plane. $m$, median; for definition of 'binocular' see text

Figure 9 shows the distribution of fixation angles (see Methods) during the initial fixation period. The diagram represents measurements from four birds. In each bird, about 80 fixation angles were measured. The median values on the right and left hemifields are $60.26^{\circ}$ and $60.45^{\circ}$, respectively. This fits in very well with the measurements of the monocular visual field, where the highest pupillary index was obtained between $50^{\circ}$ and $60^{\circ}$, and with the morphological estimation of the optical axis $\left(62^{\circ}\right)$. Therefore, one can conclude that during this fixation period the grain image falls within the foveal area.

However, although the number of pecks decreases as the direction of the grain changes from $60^{\circ}$ lateral to more frontal in both hemifields, there were a certain number of binocular fixations in these experiments (for definition of binocular fixation see Methods). These binocular pecks amounted to about $13 \%$ of the whole sample. In addition to the angle, the horizontal distance from the grain to the eye was estimated for each pecking sequence (Fig. 10). Pooling all cases with lateral and frontal fixation shows that frontal fixation occurs more often at shorter distances $(1.55 \mathrm{~cm} \pm 0.4$, mean $\pm S D$ ), whereas lateral fixation correlates with larger grain distances $(2.7 \mathrm{~cm} \pm 0.75)$. The difference between the two means is highly significant according to the Student's $t$ test (two-tailed $t=$ 9.71, $P<0.0001$ ).

The number of monocular fixations with the right and left eye were counted in eight birds. As shown in Table 1, four birds had a tendency to prefer the right eye, whereas the other four tended to prefer the left eye; three birds had a significant 


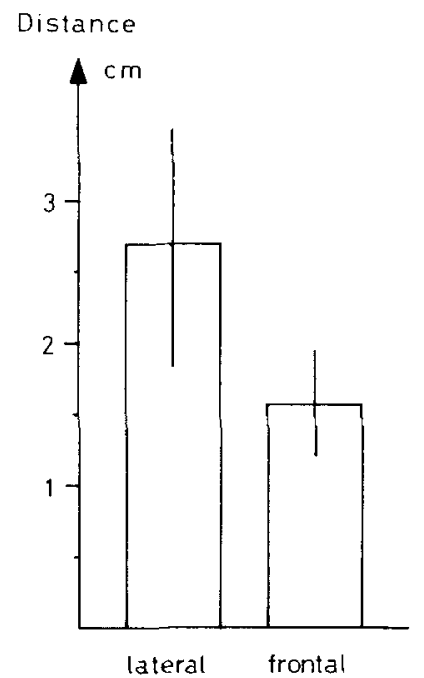

Fig. 10. Mean distances $\pm S D$ (horizontal components) between eye and grain with lateral and frontal fixation before pecking

Table 1. Number of fixations performed with the left or the right eye during one experiment in eight birds

\begin{tabular}{rccl}
\hline Bird & $\begin{array}{l}\text { Left hemifield } \\
(n)\end{array}$ & $\begin{array}{l}\text { Right hemifield } \\
(n)\end{array}$ & $P \leq$ \\
\hline S & 77 & 93 & n.s. \\
E 48 & 116 & 128 & n.s. \\
1004 & 74 & 46 & 0.01 \\
E 43 & 25 & 37 & n.s. \\
1 & 96 & 53 & 0.0004 \\
2 & 67 & 63 & n.s. \\
10 & 22 & 45 & 0.005 \\
12 & 47 & 23 & 0.004 \\
\hline
\end{tabular}

Highest values of each experiment are in italics. $P$ values are significances in a $\chi^{2}$ test
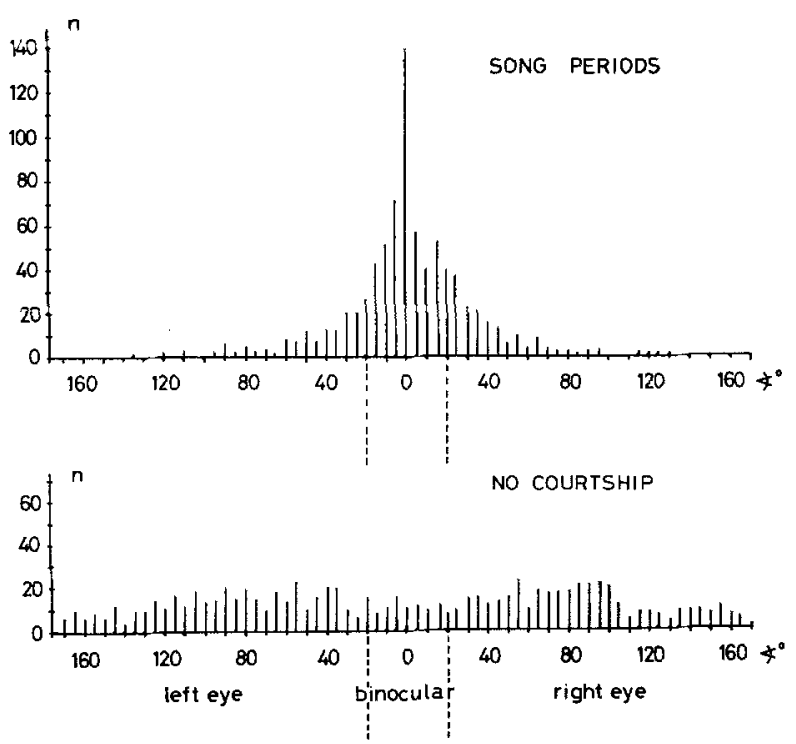

Fig. 11. Distribution of viewing angles of the male during and outside courtship song bouts. For explanation see text preference for the left eye and one had a significant preference for the right eye $\left(\chi^{2}\right.$ test, Table 1$)$. The results indicate that, at least in pecking, there is no consistent preference for one eye. If anything, there is a slight tendency to use the left eye. In addition, preliminary observations indicate that the birds sometimes change the preferred side.

\section{Male courtship behavior during singing}

As mentioned in the introduction, one aim of the study was to determine whether the males fixate the female in courtship behavior with a different part of the retina than in pecking sequences. Measurements were restricted to periods in which the bird sang the courtship song as this part of courtship was easy to detect on the tape. As a comparison sequences from the tape were chosen where the birds obviously did not show any courtship behavior. The results of the measurements are shown in Fig. 11. Surprisingly, during the courtship song the males preferred to direct their heads directly towards the female (measurements of four birds, $n=680$, Fig. 11, upper graph). This might be taken to indicate that the bird fixates the female binocularly during the courtship song. However, in the discussion an alternative explanation is offered. The control measurements during courtshipfree periods (four birds, $n=350$ ) demonstrate that the male prefers neither monocular nor binocular fixation, although there is a slight tendency to fixate monocularly at around $60^{\circ}$. During these periods males probably do not look at the female at all.

\section{Discussion}

The data demonstrate that each zebra finch eye has a large monocular visual field of nearly $170^{\circ}$ in the horizontal direction. In front of the head there is an overlap of $30^{\circ}-40^{\circ}$ between the visual fields of both eyes. In this part of the field binocular vision is possible. Caudally, the bird has a blind area of about $60^{\circ}$. The values obtained in this study are similar to those provided by Martinoya et al. (1981) and McFadden and Reymond (1985) for the pigeon and to those for the starling (Martin 1986a). In contrast, the mallard duck (Martin $1986 \mathrm{~b}$ ) has a frontal binocular overlap of only $20^{\circ}$. A caudal blind area has not been detected in these birds. The binocular field of the duck does not extend below the beak, unlike the other birds mentioned above. Martin (1986b) interprets this difference as an adaptation to feeding strategies. Whereas the duck feeds by dabbling for material which 
is strained through the bill, the other birds actively take grains or small animals with their bills and therefore have to receive information about the exact position of the tip of the beak.

The size of both the binocular overlap and the blind area depend on the eye movements of the birds. Earlier studies considered naturally occurring eye movements to be very small, e.g., in the ring dove (Friedmann 1975). However, Bloch et al. $(1981,1984)$ and Martinoya et al. (1984) have demonstrated that eyes converge substantially during feeding in the pigeon. They have further demonstrated that the eyes converge coordinately during feeding, but can be moved independently in lateral vision. The maximum amplitude of eye movements obtained in their studies was about $\pm 15^{\circ}$. This fits well with the data of Martin (1986a) from the starling. Preliminary observations in the zebra finch suggest that the eye movements are about $\pm 10^{\circ}$ to $\pm 15^{\circ}$ in birds with fixed heads, which is in accordance with the above-mentioned studies. However, due to the method of drawing the bird's attention to the microscope used here, the extreme boundaries of the binocular field were probably measured.

It seems likely that the birds use their monocular (lateral) and binocular (frontal) visual fields, at least partly, for different tasks. In the pigeon it has been demonstrated that these birds are myopic in the frontal visual field and hyperopic in the lateral field (Nye 1973; Marshall et al. 1973; Bloch and Martinoya 1978, 1982). Bloch et al. (1984) demonstrated that the mode of interaction between the two eyes is different in frontal and lateral fixation. With frontal fixation the eyes converge simultaneously when the head moves towards a grain. With lateral fixation, however, both eyes move independently. Bloch et al. (1984) concluded, as others had previously, that the frontal visual field is adapted for visually guided tasks at near distance (feeding or landing), whereas the lateral visual field is adapted for tasks at far distances (e.g., for detecting a predator).

The results presented here suggest that this is also true for zebra finches. In the experiment with the rotating drum it was demonstrated that the highest velocity able to elicit head nystagmus is lower with frontal viewing than with lateral viewing. There are two possible explanations for this. Firstly, the refraction of the eye may be similar in the frontal and lateral parts, and the sensitivity to moving stimuli may be larger in the lateral field than in the frontal due to some unknown neuronal properties of the visual system. A second explanation may be that the image of the moving pattern is not as sharp in the frontal visual field due to myopia of the eye in this region. In the latter case, the upper velocity threshold should also be lower with exposed frontal but covered lateral eye fields. Further experiments using different distances between the frontal and lateral stimuli are required to distinguish between the two possibilities. The results of most other studies (Catania 1963; Nye 1973; Bloch and Martinoya 1982; Martinoya et al. 1983 ) indicate that the second explanation is plausible but they do not exclude the first possibility.

Concerning the lateral field, all the measurements in this study show that the point of best monocular viewing is at an angle of about $50^{\circ}-65^{\circ}$ from the sagittal axis. This can be seen by the direct measurements of the optical axis (Fig. 3) as well as by the measurements of the pupillary index (Fig. 5). As indicated in Fig. 3, the fovea is located in this region. Therefore, birds which are looking monocularly would be expected preferentially to use this region of the lateral field for fixation of objects. This is indeed true for the zebra finches. The measurements of the angle of fixation before pecking clearly demonstrate that the preferred direction for monocular fixation of the grain is at about $60^{\circ}$ from the sagittal axis of the head, which corresponds to the position of the fovea.

It is rather difficult to compare these results with those of other studies. Friedman's (1975) studies on barbary doves were obviously on birds which were walking between successive pecks. He described the birds as having successive periods of head movement and stability, and he calculated the grain position for the successive, stable intervals before the birds pecked. His results demonstrate that in periods before the birds begin pecking there is a considerable amount of monocular fixation, whereas in the last two intervals before pecking fixation is mostly binocular.

Zebra finches do not walk or hop frequently during feeding; feeding bouts alternate with bouts of hopping. Therefore, the situation in this experiment may be much more comparable to experiments where pigeons are feeding from a feeder (i.e., not walking between feeding bouts). In this situation, as Goodale (1983) has described, the pigeons show two successive head stops, where they probably fixate the grain binocularly. In this study only one head stop was observed between different feeding acts in the zebra finch, this occurring at the beginning of the stereotyped movement of the beak towards the grain. However, it might be that another head stop which is shorter than $40 \mathrm{~ms}$ occurs before the birds close their eyes; this would not be detectable by the methods used here as the video 
frames were at $40-\mathrm{ms}$ intervals. If the head stop observed here corresponds to the first head stop described by Goodale (1983), the difference in the pecking behavior of zebra finches and pigeons is considerable, as the zebra finches prefer to look at the grain monocularly during this head stop, whereas pigeons fixate the grain binocularly.

Friedman (1975) further demonstrated that in the barbary dove the proportion of monocular and binocular fixations depended to a certain degree on the distance of the grain. The same tendency can be seen from these experiments in the zebra finch, as the few grains which were fixated binocularly were significantly nearer than the ones which were fixated monocularly. Friedman (1975) also showed that monocular fixation is more frequently used in cases where the birds have to differentiate between seed glued to the ground and loose seeds.

Considering the findings in other birds, the data presented in this study may indicate that the ability of the zebra finch to see binocularly is poor compared to that of other birds, e.g., the pigeon. This would explain why zebra finches have to fixate monocularly (with the foveal region) before pecking. If this really is the case, one would presume that the zebra finch does not have a pronounced second retinal area comparable to the red area of the pigeon. As mentioned above, such a structure is not detectable by inspection. However, further research is needed to decide this question.

As the whole movement of the bird's head during feeding does not seem to be very economic, it can be assumed that another explanation for this behavior is at least possible. As the zebra finches in this study were reared under conditions where they did not learn to get their grains from the floor, but received them from a cup, it is possible that the experimental situation for the bird was as strange as the situation with the glued seeds for Friedman's doves. They may therefore have fixated the grain monocularly, as the resolution of the foveal area might be better than that of the retinal area where the binocular visual field is represented.

These results suggest that zebra finches, like pigeons, have lower spatial resolution in the frontal field, at least for far distances. Therefore, it seems puzzling that males direct their heads towards the female while singing the courtship song. If they really are short-sighted in the frontal visual field, this would mean that they could not see the female sharply during singing. It may be that males do not look at the female in order to fixate her, but to present her with the best plumage pattern (see Fig. 1). However, as courtship behavior is a chain of alternate male and female responses, the male always has to have control over the reactions of the female.

This conflict may be solved by an observation of Hodos (personal communication), who has demonstrated that short-sightedness in pigeons is restricted to the lower part of the frontal visual field; parts above the plane of the beak are normally sighted. If this is true for zebra finches, the male could readily control the female's behavior during the courtship song, while presenting the optimal head coloration pattern.

In these experiments no consistent asymmetries were found concerning the use of the left or right eye in pecking or courtship behavior. At best, there was a slight tendency to use the left eye. It has been demonstrated that some birds have better discrimination abilities with the right eye (chicken, Mench and Andrew 1986; pigeon, Güntürkün 1985). However, the difference between their results and the ones presented here may be explained by the fact that in their experiments the birds could view only with one eye, whereas the birds in this study were not restricted to monocular viewing. Therefore, the fine differences in discrimination detected in their studies may not be important in the natural situation. Similarly, the results presented here on courtship behavior are different from those of Workman and Andrew (1986) for zebra finches. This may be explained by the fact that bouts of courtship song were used in the present study, whereas Workman and Andrew observed 'intercourtship bouts' and found a right-eye preference that contrasts with the findings here. This difference of fixation in different parts of the courtship chain should be resolved by future research.

Acknowledgements. I wish to thank the students of several courses on "Verhaltensphysiologische Versuche zum optischen Sinn der Vögel' for collecting the data and M. Bredenkötter for his help in some of the experiments. Also, I wish to thank Nicky Clayton for a discussion of the results, E. Geißler for preparing the figures, S. Hörnle for editing the manuscript, and R. Church and N. Clayton for improving the English. Last but not least, my thanks are due to an anonymous referee who gave helpful hints for improving the manuscript. This study was supported by grants from the Deutsche Forschungsgemeinschaft ( $\mathrm{Bi} 245 / 3$ ), the Minister für Wissenschaft und Forschung des Landes Nordrhein-Westfalen, and a research grant from the University of Bielefeld.

\section{References}

Bischof HJ (1985) Der Anteil akustischer Komponenten an der Auslösung der Balz männlicher Zebrafinken (Taeniopygia guttata castanotis). J Ornithol 126:273-279 
Bloch S, Martinoya C (1978) Comparison of frontal and lateral visual acuity of the pigeon under behavioral fixation. $J$ Physiol (Lond) 278:49 P

Bloch S, Martinoya C (1982) Comparing frontal and lateral viewing in the pigeon. I. Tachistoscopic visual acuity as a function of distance. Behav Brain Res 5:231-244

Bloch S, Martinoya C, Rivaud S (1981) Eye movements in birds: participation in binocular fixation and visual pursuit. J Physiol (Lond) 320:20-21

Bloch S, Riveaud S, Martinoya C (1984) Comparing frontal and lateral viewing in the pigeon. III. Different patterns of eye movements for binocular and monocular fixation. Behav Brain Res 13:173-182

Catania AC (1963) Techniques for the control of monocular and binocular viewing in the pigeon. J Exp Anal Behav 6:627-629

Donovan WJ (1978) Structure and function of the pigeon visual system. Physiol Psychol 6:403-437

Emmerton J (1983a) Functional morphology of the visual system. In: Abs M (ed) Physiology and behavior of the pigeon. Academic, London, pp 221-241

Emmerton J (1983b) Vision. In: Abs M (ed) Physiology and behavior of the pigeon. Academic, London, pp 245-266

Friedman MB (1975) How birds use their eyes. In: Wright P, Caryl P, Vowles DM (eds) Neural and endocrine aspects of behavior in birds. Elsevier, Amsterdam, pp 181-204

Goodale MA (1983) Visually guided pecking in the pigeon ( $\mathrm{Col}-$ umba livia). Brain Behav Evol 22:22-41

Güntürkün O (1985) Lateralization of visually controlled behavior in pigeons. Physiol Behav 34:575-577

Immelmann K (1959) Experimentelle Untersuchungen über die biologische Bedeutung artspezifischer Merkmale beim Zebrafinken (Taeniopygia guttata castanotis GOULD). Zool Jb $86: 438-593$
Marshall J, Mellerio J, Palmer DA (1973) A schematic eye for the pigeon. Vision Res 13:2449-2453

Martin GR (1986a) The eye of a passeriform bird, the European starling (Sturnus vulgaris): eye movement amplitude, visual fields and schematic optics. J Comp Physiol A 159:545-557

Martin GR (1986b) Total panoramic vision in the mallard duck, Anas platyrhynchos. Vision Res 26:1303-1305

Martinoya C, Rey J, Bloch S (1981) Limits of the pigeon's binocular field and direction for best binocular viewing. Vision Res $21: 1197-1200$

Martinoya C, Riveaud S, Bloch S (1983) Comparing frontal and lateral viewing in the pigeon. II. Velocity thresholds for movement discrimination. Behav Brain Res 8:375385

Martinoya C, Le Houezec J, Bloch S (1984) Pigeon's eyes converge during feeding: evidence for frontal binocular fixation in a lateral-eyed bird. Neurosci Lett 45:335-339

McFadden SA, Reymond L (1985) A further look at the binocular visual field of the pigeon (Columba livia). Vision Res 25:1741-1746

Mench JA, Andrew RJ (1986) Lateralization of a food search task in the domestic chick. Behav Neural Biol 46:107-114

Meyer DB (1977) The avian eye and its adaptations. In: Crescitelli $F$ (ed) The visual system in vertebrates. (Handbook of sensory physiology, vol VII/5) Springer, Berlin Heidelberg New York, pp 549-611

Morris D (1954) The reproductive behavior of the zebra finch. Behaviour 6:271-322

Nye PW (1973) On the functional differences between frontal and lateral visual fields of the pigeons. Vision Res 13:559-574

Workman L, Andrew RJ (1986) Asymmetries of eye use in birds. Anim Behav 34:1582-1585 